\title{
Integrating nanoscale technologies with cryogenics: a step towards improved biopreservation
}

\author{
"New horizons in cryobiology could be explored by nanotechnology, which has \\ revolutionized multiple fields in science. Some of the advances in materials science and \\ nanotechnology ... can also be adapted to cryopreservation..."
}

\section{KEYWORDS: cryopreservation - cryoprotectant agent $\approx$ minimum volume vitrification - nanotechnology}

At the beginning of the 20th century, successful preservation methods for cells and tissues were developed with new insights into biology and physics. In nature, we have already seen interesting examples of biopreservation. For instance, species such as water-bears (e.g., Tardigrada) and distinct frog types (e.g., Rana sylvatica) can survive in extremely cold temperatures by replacing the water in their bodies with highly concentrated glucose, which serves as an antifreezing agent [1]. These species can keep their metabolism at minimal levels under belowfreezing conditions. Inspired by nature, the holy grail of cryopreservation is to stop or slow down cellular and biological activity by bringing cells down to low temperatures, and later, to be able to restore function at physiological temperatures [2].

The major interest in biopreservation is driven by advances in medicine and biology, along with emerging clinical needs. Various cell types, including stem cells, embryonic cells, genetically modified cells, cancer cells, hematopoietic cells, germ cells and adult stromal cells are routinely cryopreserved for both research and clinical purposes. The scope of cryopreservation covers preservation of fertility, cell therapies, reproductive medicine, regenerative medicine, stem cell research, blood preservation and handling of organs prior to transplantation. In addition to these applications, long-term preservation of animal and plant cells as well as the genetic material of endangered species are also in the scope of cryopreservation.

Although there has been significant progress in biopreserving various cell types, the existing procedures cause cryo-injury, such as dehydration of cells, loss of cell membrane integrity, alterations in functionality by potential disturbance of the cytoskeleton and apoptosis or changes in the extracellular matrix properties of tissues [3,4]. Improving cryopreservation outcomes by overcoming some of the existing limitations can be achieved by better understanding what really happens at the micro- and nano-scales during the freezing processes. For instance, at temperatures below $4^{\circ} \mathrm{C}$, water density decreases as its volume increases, while at sub-zero temperatures, water crystallizes [5]. Extensive crystallization and volume expansion may cause cryo-injury to cells. To address these biopreservation challenges, two methods have been traditionally employed - slow and fast freezing [6]. Both of these methods may lead to cell damage during loading/unloading of the cryoprotectant agents (CPAs), freezing and thawing steps [7]. To address these issues, various types of CPAs have been employed to decrease the freezing point, create an osmotic buffer and increase solute concentration, and thereby minimize cryo-injury. The most commonly used CPAs are dimethylsulfoxide, 1,2-propanediol, glycerol, ethylene glycol, trehalose, proline, mannitol and sucrose [8]. Although CPAs are used as preserving reagents, they can be toxic and have negative effects on cell function.

The slow freezing method is the most commonly used cryopreservation technique in clinics and research laboratories today. Slow freezing first replaces the water within the cytoplasm with CPAs, which minimizes cell damage and adjusts the cooling rate in accordance with the permeability of the cell membrane. Typically, mammalian cells are frozen at a rate of $1{ }^{\circ} \mathrm{C} / \mathrm{min}$ at CPA concentrations as low as $1.5 \mathrm{M}$ [6]. Additionally, in most clinical practices, cell supply is limited or the cell population is rare (e.g., oocytes, autologous stem cells from bone

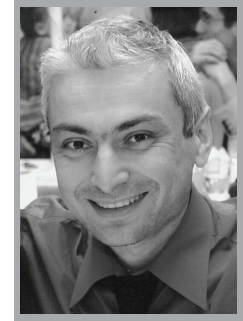

Sinan Guven

Division of Biomedical Engineering, Bio-Acoustic MEMS in Medicine (BAMM) Laboratory, Department of Medicine, Brigham \& Women's Hospital, Harvard Medical School, MA, USA

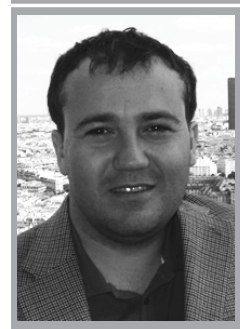

Utkan Demirci

Author for correspondence: Division of Biomedical Engineering, Bio-Acoustic MEMS in Medicine (BAMM) Laboratory, Department of Medicine, Brigham \& Women's Hospital, Harvard Medical School, MA, USA

and Harvard-MIT Health Sciences and Technology, Cambridge, MA, USA udemirci@rics.bwh.harvard.edu 
marrow and cord blood stromal cells). Therefore, a significant loss in the viability of cryopreserved cells is not desirable.

\section{"Fully validated, closed, automated and affordable systems are needed for cryopreservation of cells for clinical applications."}

Vitrification is an alternative cryopreservation technique that addresses loss in cell viability resulting from ice crystal formation through a rapid phase transition from liquid to a glasslike solid $[9,10]$. Achieving successful vitrification requires up to sixfold higher CPA concentrations compared with that of slow freezing and more rapid cooling rates of up to $250,000^{\circ} \mathrm{C} / \mathrm{min}$ [11]. To achieve higher heat transfer rates and to minimize CPA levels, minimum sample volume-based vitrification techniques have been developed [6,9]. Minimum sample volume approaches are enabled by technological advances that have allowed manipulation of cells in micro- and nano-scale volumes. For instance, microfluidic channels have been used to gradually load and unload cells with CPAs, minimizing osmotic shock [12]. Our capability to encapsulate cells in nanoliter droplets of various types of fluids including cryoprotectants and hydrogels, along with nanomaterials such as magnetic nanoparticles, enables new capabilities to manipulate cells and biofluids [13-15]. For instance, an ejector-based system has been developed to generate nanoliter droplets continuously and at high flow rates, which can then be vitrified by ultra-rapid cooling upon direct ejection into liquid nitrogen [2] or onto a thin film that is later immersed into liquid nitrogen [8]. This approach has been successfully applied to the cryopreservation of mouse oocytes with functional studies of post-thawed cells for parthenogenetic development [16]. This method allows lower CPA levels for vitrification and reduces the osmotic stress on cells during CPA loading before vitrification and unloading after thawing.

The most common vitrification techniques include straw, open pulled straw, glass or quartz capillary, cryo-loop and electron microscopy grid $[6,9,11]$. One of the challenges of vitrification methods is limited throughput to biopreserve large sample volumes. Up-scaling a vitrification system while maintaining high efficiency has been recently presented with the nanoliter droplet vitrification method using an array of droplet ejectors [8]. This method has also been applied to cryopreserve larger volumes of cells such as red blood cells. For instance, demand for biopreserved blood is critical during natural disasters and military conflicts. While the advantages of vitrification using minimum volume methods have been presented, there are some bottlenecks in the translation of these technologies to successful and widespread clinical applications. Fully validated, closed, automated and affordable systems are needed for cryopreservation of cells for clinical applications.

\section{Future goals in cryopreservation using nanotechnology}

The rapid evolution of science has increased public expectations for solutions addressing today's challenging questions. Current technologies enable us to understand nature and mimic underlying mechanisms to solve biomedical challenges. This pursuit has resulted in biomimetic approaches that have brought novel and elegant ideas for targeting solutions. New horizons in cryobiology could be explored by nanotechnology, which has revolutionized multiple fields of science. Some of these advances in materials science and nanotechnology, such as nanodirectional surfaces that can manipulate droplets carrying biological cargo, can also be adapted to cryopreservation [17,18]. Freezing and manipulating cells encapsulating nanoliter droplets on super-hydrophobic nanorough surfaces is one of the potential ways of manipulating droplets, as minimal volume technologies offer potential solutions to the current challenges of cryobiology. Nanoengineered superhydrophobic surfaces can be designed, mimicking the architecture of lotus leaves and rose petals [19]. High contact angles between surfaces and nanoliter droplets could enable control over the shape and size of droplets being manipulated for biopreservation. Bio-inspired approaches in cryopreservation could also lead to the development of mechanisms and materials mimicking nature, such as extremophilic microorganisms (i.e., psychrobacter, arthrobacter and hyperthermophile genus). These organisms can resist extreme temperatures because of their peptidoglycanbased cell wall, which varies from 2 to $60 \mathrm{~nm}$ in thickness $[20,21]$. This cell wall protects the bacteria from external factors, changes in osmotic balance and provides mechanical integrity. Most microbiological studies target the removal of the bacteria cell wall by inhibiting its synthesis or degrading it enzymatically or chemically [22]. The protective features of a peptidogylcan cell wall can be adapted to preserve mammalian cells. Cell envelopes of nanometer thickness can help to enhance traditional CPAs and lead to advances in cryopreservation. Similarly, novel bio-inspired 
CPAs that are not toxic to cells can be adapted for cryopreservation applications. There are many examples such as ectoine, a widely used skin protecting agent that was first derived from bacteria and is now a promising natural cell protectant [23]. In summary, bio-inspired nanotechnologies promise novel solutions to fulfill needs of cryopreservation in medicine.

\section{Acknowledgements}

This work was performed at the Bio-Acoustic MEMS in Medicine Laboratories at the HST-BWH Center for Bioengineering, Harvard Medical School.

\section{Financial \& competing interests disclosure}

U Demirci acknowledges that this material is based in part upon work supported by the National Science Foundation under NSF CAREER Award Number 1150733, NIH R01EB015776-01A1 and NIH R21HL095960-02. The authors have no other relevant affiliations or financial involvement with any organization or entity with a financial interest in or financial conflict with the subject matter or materials discussed in the manuscript apart from those disclosed.

No writing assistance was utilized in the production of this manuscript.

\section{References}

1 Guidetti R, Rizzo AM, Altiero T, Rebecchi L. What can we learn from the toughest animals of the Earth? Water bears (Tardigrades) as multicellular model organisms in order to perform scientific preparations for lunar exploration. Planet. Space Sci. doi:10.1016/ j.pss.2012.05.021 (2012) (Epub ahead of print).

2 Demirci U, Montesano G. Cell encapsulating droplet vitrification. Lab Chip 7(11), 1428-1433 (2007).

3 Leibo SP, Pool TB. The principal variables of cryopreservation: solutions, temperatures, and rate changes. Fertil. Steril. 96(2), 269-276 (2011).

4 Ragoonanan V, Hubel A, Aksan A. Response of the cell membrane-cytoskeleton complex to osmotic and freeze/thaw stresses. Cryobiology 61(3), 335-344 (2010).

5 Chian R-C, Quinn P. Cryobiology: an Overview Fertility Cryopreservation. Cambridge University Press, Cambridge, UK, 1-9 (2010).

6 Zhang X, Catalano PN, Gurkan UA, Khimji I, Demirci U. Emerging technologies in medical applications of minimum volume vitrification. Nanomedicine (Lond.) 6(6), 1115-1129 (2011).

7 Farrant J, Molyneux P, Hasted JB, Meares P, Echlin P. Water transport and cell survival in cryobiological procedures [and discussion].

\section{Phil. Trans. R. Soc. Lond. B 278(959), 191-} 205 (1977).

8 Samot J, Moon S, Shao L et al. Blood banking in living droplets. PLoS ONE 6(3), e17530 (2011).

9 Arav A, Yavin S, Zeron Y, Natan D, Dekel I, Gacitua H. New trends in gamete's cryopreservation. Mol. Cell. Endocrinol. 187(1-2), 77-81 (2002).

10 Fahy GM, Macfarlane DR, Angell CA, Meryman HT. Vitrification as an approach to cryopreservation. Cryobiology 21(4), 407-426 (1984).

11 Risco R, Elmoazzen H, Doughty M, He X, Toner M. Thermal performance of quartz capillaries for vitrification. Cryobiology 55(3), 222-229 (2007).

12 Song YS, Moon S, Hulli L, Hasan SK, Kayaalp E, Demirci U. Microfluidics for cryopreservation. Lab Chip 9(13), 1874-1881 (2009).

13 Xu F, Inci F, Mullick O et al. Release of magnetic nanoparticles from cellencapsulating biodegradable nanobiomaterials. ACS Nano 6(8), 6640-6649 (2012).

14 Moon S, Kim Y-G, Dong L et al. Drop-ondemand single cell isolation and total RNA analysis. PLoS ONE 6(3), e17455 (2011).

$15 \mathrm{Xu}$ F, Wu C-aM, Rengarajan V et al. Threedimensional magnetic assembly of microscale hydrogels. Adv. Mater. 23(37), 4254-4260 (2011).
16 Zhang X, Khimji I, Shao L et al. Nanoliter droplet vitrification for oocyte cryopreservation. Nanomedicine (Lond.) 7(4), 553-564 (2012).

17 Sekeroglu K, Gurkan UA, Demirci U, Demirel MC. Transport of a soft cargo on a nanoscale ratchet. Appl. Phys. Lett. 99(6), 063703-063703-3 (2011).

18 Malvadkar NA, Hancock MJ, Sekeroglu K, Dressick WJ, Demirel MC. An engineered anisotropic nanofilm with unidirectional wetting properties. Nat. Mater. 9(12), 1023-1028 (2010).

19 Park YM, Gang M, Seo YH, Kim BH. Artificial petal surface based on hierarchical micro- and nanostructures. Thin Solid Films 520(1), 362-367 (2011).

20 Vollmer W, Blanot D, De Pedro MA. Peptidoglycan structure and architecture. FEMS Microbiol. Rev. 32(2), 149-167 (2008).

21 Cavicchioli R, Siddiqui KS, Andrews D, Sowers KR. Low-temperature extremophiles and their applications. Curr. Opin. Biotechnol. 13(3), 253-261 (2002).

22 Kohanski MA, Dwyer DJ, Collins JJ. How antibiotics kill bacteria: from targets to networks. Nat. Rev. Micro. 8(6), 423-435 (2010).

23 Graf R, Anzali S, Buenger J, Pfluecker F, Driller $\mathrm{H}$. The multifunctional role of ectoine as a natural cell protectant. Clin. Dermatol. 26(4), 326-333 (2008). 